\title{
Correspondence between Minkowski and de Sitter Quantum Field Theory
}

\author{
Marco Bertola ${ }^{\mathrm{a}}$, Vittorio Gorini ${ }^{\mathrm{b}}$, Ugo Moschella ${ }^{\mathrm{b}, \mathrm{c}}$, Richard Schaeffer ${ }^{\mathrm{c}}$
}

July 3,2018

\author{
a SISSA, v. Beirut 2-4, 34014 Trieste \\ b Dipartimento di Scienze Matematiche Fisiche e Chimiche, \\ Via Lucini 3, 22100 Como and INFN sez. di Milano, Italy \\ c Service de Physique Théorique, C.E. Saclay, 91191 Gif-sur-Yvette, France
}

\begin{abstract}
In this letter we show that the "preferred" Klein-Gordon Quantum Field Theories (QFT's) on a $d$-dimensional de Sitter spacetime can be obtained from a KleinGordon QFT on a $(d+1)$-dimensional "ambient" Minkowski spacetime satisfying the spectral condition and, conversely, that a Klein-Gordon QFT on a $(d+1)$ dimensional "ambient" Minkowski spacetime satisfying the spectral condition can be obtained as superposition of $d$-dimensional de Sitter Klein-Gordon fields in the preferred vacuum. These results establish a correspondence between QFT's living on manifolds having different dimensions. The method exposed here can be applied to study other situations and notably QFT on Anti de Sitter spacetime.
\end{abstract}

The study of the relations between Quantum Field Theories (QFT's) in different dimensions has come recently to the general attention. Some of the most interesting and intriguing developments of QFT and string theory, like Maldacena's ADS/CFT conjecture [1, 2] and t'Hooft's [3] and Susskind's [4] holographic principle, seem to indicate that relations of this kind are going to play a fundamental role in understanding QFT and string theory.

In this letter we point out a relation that exists between Minkowski QFT and de Sitter QFT in one dimension less. We show that the "preferred" [5, 6] de Sitter Klein-Gordon field of squared mass $\lambda$ arises by averaging in a well-defined sense an ordinary KleinGordon field of mass $M$ living in the Minkowski ambient spacetime and, vice versa, the Klein-Gordon field in the ambient Minkowski spacetime can be obtained by superposing fields in the lower dimensional de Sitter manifold. 
The idea that QFT's on the de Sitter manifold can be obtained by restriction from the ambient spacetime is of course not new, but it has been of little use in the standard coordinate approach to de Sitter field theories.

Recently, however, it has been shown [7, [] that the well-known [0] thermal properties of the de Sitter Klein-Gordon fields in the "preferred" vacuum, are linked to certain analyticity properties of the correlation functions; these properties are precisely obtained by restriction to the de Sitter manifold of the analyticity properties of the (general) correlation functions in the ambient spacetime, which hold when the corresponding QFT satisfies the energy-momentum spectral condition [9].

This idea has then been pushed further and it has become possible to show that the thermal interpretation can be established also for interacting de Sitter field theories [10].

In this letter we take one step more by showing how a Klein-Gordon Minkowski field in the Wightman vacuum gives rise to a Klein-Gordon field on the de Sitter spacetime in the "preferred" thermal vacuum, giving a further argument in favour of the adjective "preferred". Indeed, as it is well known, there are in general infinitely many inequivalent vacua corresponding to a certain QFT and one needs criteria to select the physically meaningful ones. This is true already for Minkowski QFT but, in this case, one has strong physical criteria to select among the vacua. The situation is more difficult when considering QFT's on a curved background, but several criteria have been established also in this case (many of them however work only for linear field theories). The "preferred" vacuum for de Sitter Klein-Gordon fields has been shown to satisfy many of such criteria, such as the Hadamard condition [11. Here we prove that, actually, the "preferred" KleinGordon de Sitter QFT's can be directly obtained by any massive or massless Wightman Klein-Gordon QFT in the ambient spacetime. There is however a restriction on the mass of the fields that can be obtained this way. In particular when working with a $d$ dimensional de Sitter hyperboloid of unit radius we can construct de Sitter Klein-Gordon fields whose mass is greater or equal to $(d-1) / 2$.

The existence of such a link gives also a quantitative support to the idea that a thermal effect on a curved manifold can be looked at as an Unruh effect in a higher (flat) dimensional spacetime [12, 13].

Let therefore $\mathcal{M}=\left\{X \in \mathbb{M}^{d+1}: \eta_{\mu \nu} X^{\mu} X^{\nu}<0\right\}$ be the manifold of events which are spacelike w.r.t. a chosen event (which is taken as the origin of a frame) of a $(d+1)$ dimensional Minkowski space-time $\mathbb{M}^{d+1}$. Coordinates of $\mathbb{M}^{d+1}$ are denoted by $\left\{X^{\mu}\right\}$, $\mu=0, \ldots, d$. $\mathcal{M}$ is foliated by a family of $d$-dimensional de Sitter spacetimes identified with the hyperboloids

$$
\mathcal{Y}_{R}=\left\{\eta_{\mu \nu} X^{\mu} X^{\nu}=\left(X^{0}\right)^{2}-(\vec{X})^{2}=-R^{2}\right\} .
$$

As a topological manifold, $\mathcal{M}=\mathbb{R}^{+} \times \mathcal{Y}$, where $\mathbb{R}^{+}$is the positive real half-line with coordinate $R$ and $\mathcal{Y}=\mathcal{Y}_{1}$ is the $d$-dimensional de Sitter spacetime with radius $R=1$. Points of $\mathcal{Y}$ are denoted by $y$ (i.e. $y^{2}=-1$ ).

We can therefore assign to an event of $\mathcal{M}$ coordinates $(R, y)$ so that $X=R y$ and $y^{2}=-1$. The Minkowskian metric of $\mathcal{M}$ can consequently be rewritten as follows:

$$
d s^{2}=-d R^{2}+R^{2} d s_{\mathcal{Y}}^{2},
$$


where $d s_{\mathcal{Y}}^{2}$ is the de Sitter metric of $\mathcal{Y}$ obtained as restriction of the Minkowski metric of the ambient space.

$\mathcal{M}$ is a globally hyperbolic manifold where quantum field theory can be formulated [11]. Let us therefore consider a canonical quantum field $\widehat{\Phi}$ on $\mathcal{M}$ satisfying the KleinGordon equation $\left(\square+M^{2}\right) \widehat{\Phi}(X)=0$ (in the following we will consider the massive case $M>0$; the massless case can be obtained by a limit procedure but can also be studied directly, with a considerable simplification of the formulae), and let us also consider the corresponding equation for the modes $\Phi(X)$. By separating the variables as in the metrics we write $\Phi(X)=\theta_{\lambda}(R) \varphi_{\lambda}(y)$ and we are led to the following equations:

$$
\begin{aligned}
& (\square \mathcal{Y}+\lambda) \varphi(y)=0 \\
& R^{2}\left(\partial_{R}^{2}+\frac{d}{R} \partial_{R}-M^{2}\right) \theta_{\lambda}(R)=-\lambda \theta_{\lambda}(R) .
\end{aligned}
$$

Let us consider in particular the equation (3) for the radial modes $\theta_{\lambda}$. The operator appearing at the L.H.S. is self-adjoint (on a suitable domain) w.r.t. the following Hilbert product:

$$
(\theta, \eta)=\int_{\mathbb{R}^{+}} \bar{\theta}(R) \eta(R) R^{d-2} d R
$$

By means of the transformation $\theta(R)=R^{\frac{1-d}{2}} f(R)$ and the rescaling $\rho=M R$, which together are particular instances of the so called 'Lommel's transformation', Eq. (3) is turned into the modified Bessel's equation. By further introducing the variable $x=$ $\log \rho=$, we finally obtain the following equation (the prime means derivative w.r.t. $x$ ):

$$
-f_{\lambda}^{\prime \prime}+\left(e^{2 x}-\nu^{2}\right) f_{\lambda}=0 \quad \text { with } \quad \nu=\nu(\lambda)=\sqrt{\lambda-\frac{(d-1)^{2}}{4}} .
$$

We have thus obtained the Schrödinger problem for a particle in a one-dimensional potential $e^{2 x}$ with eigenvalue $\nu^{2}$; this problem is to be studied in the standard Hilbert space $L^{2}(\mathbb{R})$. The spectrum is nondegenerate and coincides with the positive real line; this implies that $4 \lambda>(d-1)^{2}$.

The solutions which have the correct asymptotic behaviour at $x=\infty$ are the modified Bessel functions $K_{i \nu}\left(e^{x}\right)$ [14]. These modes are real, and their asymptotic behaviour near $x=-\infty$ is the following:

$$
K_{i \nu}\left(e^{x}\right) \simeq-\left[\frac{\pi \nu}{\sinh (\pi \nu)}\right]^{\frac{1}{2}} \frac{\sin [(x-\log (2)) \nu+\arg (\Gamma(1+i \nu))]}{\nu}
$$

comparison with the free Schrödinger waves gives

$$
\int_{\mathbb{R}} K_{i \nu}\left(e^{x}\right) K_{i \nu^{\prime}}\left(e^{x}\right) d x=\frac{\pi^{2}}{\sinh (\pi \nu)} \delta\left(\nu^{2}-\nu^{\prime 2}\right)=N_{\lambda}^{-2} \delta\left(\lambda-\lambda^{\prime}\right) .
$$

In terms of the original variable $R$ we therefore obtain the following normalized generalised eigenfunctions

$$
\theta_{\lambda}(R)=N_{\lambda} R^{\frac{1-d}{2}} K_{i \nu}(M R)
$$


The orthonormality and completeness conditions for these modes read

$$
\begin{aligned}
& \int_{\mathbb{R}^{+}} \theta_{\lambda}(R) \theta_{\lambda^{\prime}}(R) R^{d-2} \mathrm{~d} R=\delta\left(\lambda-\lambda^{\prime}\right) \\
& \int_{\frac{(d-1)^{2}}{4}}^{\infty} d \lambda \theta_{\lambda}(R) \theta_{\lambda}\left(R^{\prime}\right)=R^{-(d-2)} \delta\left(R-R^{\prime}\right)
\end{aligned}
$$

We now introduce the fields $\widehat{\varphi}_{\lambda}(y)$ on the de Sitter manifold $\mathcal{Y}$ by smearing the field $\widehat{\Phi}$ with the complete set of radial modes (8):

$$
\widehat{\varphi}_{\lambda}(y)=\int_{\mathbb{R}^{+}} \widehat{\Phi}(X) \bar{\theta}_{\lambda}(R) R^{d-2} d R .
$$

Our main result is that the field $\widehat{\varphi}_{\lambda}(y)$ is a Klein-Gordon field on the de Sitter manifold in the "preferred" (also called Euclidean or Bunch-Davies) vacuum state. In precise terms, the Minkowski vacuum expectation values (v.e.v.) of the fields $\widehat{\varphi}_{\lambda}(y)$ are given by

$$
W_{\lambda, \lambda^{\prime}}\left(y, y^{\prime}\right) \equiv\left\langle\Omega\left|\widehat{\varphi}_{\lambda}(y) \widehat{\varphi}_{\lambda^{\prime}}\left(y^{\prime}\right)\right| \Omega\right\rangle=\delta\left(\lambda-\lambda^{\prime}\right) W_{\lambda}\left(y, y^{\prime}\right)
$$

where $W_{\lambda}$ is the "preferred" two-point function of de Sitter Klein-Gordon field in dimension $d$ [8]. In particular, the fields $\widehat{\varphi}_{\lambda}$ have zero correlation (and hence commute) for different values of the square mass $\lambda$.

We now will give an argument to prove the result by first of all deriving an explicit expression for $W_{\lambda, \lambda^{\prime}}\left(y, y^{\prime}\right)$ (eq. 15 below) . Let us rewrite the v.e.v. appearing at the LHS of Eq. (12) by using the momentum representation of the two-point function of the field $\widehat{\Phi}(X)$ :

$$
\begin{aligned}
& W_{\lambda, \lambda^{\prime}}\left(y, y^{\prime}\right)= \\
& =\int_{0}^{\infty} \frac{d R}{R} R^{d-1} \theta_{\lambda}(R) \int_{0}^{\infty} \frac{d R^{\prime}}{R^{\prime}} R^{\prime d-1} \theta_{\lambda^{\prime}}\left(R^{\prime}\right) \int \frac{\mathrm{d}^{d+1} P}{(2 \pi)^{d}} \delta\left(P^{2}-M^{2}\right) \Theta\left(P_{0}\right) e^{-i P\left(X-X^{\prime}\right.}(13)
\end{aligned}
$$

In this expression we insert the parametrisations $X=R y$ and $X^{\prime}=R^{\prime} y^{\prime}$ and introduce the vector $\alpha$ defined by the relation $M \alpha=P$, where $P$ is on the mass shell; $\alpha$ is therefore on the unit shell $\alpha^{2}=1, \alpha_{0}>0$.

By exchanging the order of integration we are led to the following integrals ([14], Vol II, Eq. (7.8.5)):

$$
\begin{aligned}
& \varphi_{\lambda}(y, \alpha)=\varphi_{\lambda}(y \cdot \alpha)=M^{\frac{d-1}{2}} \int_{0}^{\infty} e^{i y \cdot \alpha M R} \theta_{\lambda}(R) R^{d-1} \frac{d R}{R}= \\
& =\sqrt{\frac{\pi}{2}} N_{\lambda} \Gamma\left(\frac{d-1}{2}-i \nu\right) \Gamma\left(\frac{d-1}{2}+i \nu\right)\left((-i y \cdot \alpha)^{2}-1\right)^{\frac{2-d}{4}} P_{-\frac{1}{2}-i \nu}^{\frac{2-d}{2}}(-i y \cdot \alpha)
\end{aligned}
$$

where the factor $M^{\frac{d-1}{2}}$ has been inserted for convenience so that the function is dimensionless.

The functions $\varphi_{\lambda}(y, \alpha)$ are a new set of plane waves on de Sitter manifold i.e. are (global) 
modes satisfying the de Sitter Klein-Gordon equation (2) whose phase is constant on planes; $\alpha$ plays the role of wave-vector and $P$ is an associated Legendre function [14. The integral appearing in the definition of these waves is well defined at both extrema provided $|\Im(\nu)|<\frac{d-1}{2}$.

We are finally led to consider the following expression:

$$
W_{\lambda, \lambda^{\prime}}\left(y, y^{\prime}\right)=\int \frac{\mathrm{d}^{d+1} \alpha}{(2 \pi)^{d}} \delta\left(\alpha^{2}-1\right) \Theta\left(\alpha_{0}\right) \bar{\varphi}_{\lambda}(y, \alpha) \varphi_{\lambda^{\prime}}\left(y^{\prime}, \alpha\right) .
$$

This formula coincides with the "preferred" two-point function of the de Sitter KleinGordon field in dimension $d$ (see Eq. 12), giving at the same time a new integral representation for it. The actual full proof of this claim is somewhat involved and will be given elsewhere [15].

We can however illustrate the result in the simplest case $d=1$ where things are easier. In this case the de Sitter spacetime has only "time" and no "space" : it can be visualised as the two branches of an hyperbola in the two-dimensional Minkowski spacetime; these represent the world-lines of two uniformly accelerated observers.

The plane waves $\varphi_{\lambda}$ reduce here to ordinary trigonometric functions in the rectifying parameter of the hyperbola.

To carry out the computation of the integral (15) we parametrise $\alpha=\left(\begin{array}{c}\cosh (s) \\ \sinh (s)\end{array}\right)$ and $y(t)=\left(\begin{array}{c}\sinh (t) \\ \pm \cosh (t)\end{array}\right) ; t$ is the proper time of the 'freely falling' observer in the one-dimensional de Sitter spacetime (the same observer is actually subject to a constant acceleration when regarded from the Minkowskian ambient space). By taking the two points on the same branch of the hyperbola (say, the right one) we promptly find $\alpha \cdot y(t)=$ $\sinh (s+t)$. The measure $\mathrm{d}^{2} \alpha \delta\left(\alpha^{2}-1\right) \Theta\left(\alpha_{0}\right)$ becomes simply $\frac{1}{2} \mathrm{~d} s$.

A second step exploits the analyticity properties of the Minkowskian Wightman function and of our plane waves by shifting the two times $t, t^{\prime}$ by an imaginary part $t \mapsto t-i \pi / 2$ and $t^{\prime} \mapsto t^{\prime}+i \pi / 2$. It follows that

$$
\begin{aligned}
& \int_{\mathbb{R}} \frac{d s}{2(2 \pi)} \int \frac{d R}{R} \int \frac{d R^{\prime}}{R^{\prime}} e^{-i \alpha(s) \cdot\left(y(t)-y\left(t^{\prime}\right)\right)} \theta_{\lambda}(R) \theta_{\lambda^{\prime}}\left(R^{\prime}\right) \stackrel{t}{t^{\prime}} \mapsto \underline{t} \stackrel{\mapsto \pi / 2}{t^{\prime}+i \pi / 2} \\
& \mapsto \int_{\mathbb{R}} \frac{d s}{2(2 \pi)} \int \frac{d R}{R} \int \frac{d R^{\prime}}{R^{\prime}} e^{-M\left(R \cosh (s+t)+R^{\prime} \cosh \left(s+t^{\prime}\right)\right)} \theta_{\lambda}(R) \theta_{\lambda^{\prime}}\left(R^{\prime}\right)= \\
& =N_{\lambda} N_{\lambda^{\prime}} \int_{\mathbb{R}} \frac{d s}{2(2 \pi)}|\Gamma(i \nu)|^{2} \cos (\nu(s+t))\left|\Gamma\left(i \nu^{\prime}\right)\right|^{2} \cos \left(\nu^{\prime}\left(s+t^{\prime}\right)\right)= \\
& =\frac{1}{4} N_{\lambda} N_{\lambda^{\prime}}|\Gamma(i \nu)|^{2}\left|\Gamma\left(i \nu^{\prime}\right)\right|^{2} \delta\left(\nu-\nu^{\prime}\right) \cos \left(\nu\left(t-t^{\prime}\right)\right)= \\
& =\frac{1}{4 \nu^{2} \sinh (\pi \nu)} \delta\left(\nu-\nu^{\prime}\right) \cos \left(\nu\left(t-t^{\prime}\right)\right)=\delta\left(\lambda-\lambda^{\prime}\right) \frac{\cos \left(\nu\left(t-t^{\prime}\right)\right)}{2 \nu \sinh (\pi \nu)}
\end{aligned}
$$

Returning to the Minkowski spacetime from the correct tubular domains $(t \mapsto t+i \pi / 2$ 
and $\left.t^{\prime} \mapsto t^{\prime}-i \pi / 2\right)$ we obtain the result

$$
\left\langle\Omega\left|\widehat{\varphi}_{\lambda}(y(t)) \widehat{\varphi}_{\lambda^{\prime}}\left(y\left(t^{\prime}\right)\right)\right| \Omega\right\rangle=\delta\left(\lambda-\lambda^{\prime}\right) \frac{\cos \left(\nu\left(t-t^{\prime}+i \pi\right)\right)}{2 \nu \sinh (\pi \nu)}=\delta\left(\lambda-\lambda^{\prime}\right) W_{\lambda}\left(y(t), y\left(t^{\prime}\right)\right) .
$$

This two-point function correspond to the "preferred" one for de Sitter Klein-Gordon quantum field as given in [8] as well as to that of a quantum harmonic oscillator in a thermal state at the inverse temperature $2 \pi$. Indeed, the quantum Klein-Gordon field on a one-dimensional spacetime corresponds to a single quantum harmonic oscillator in the Heisenberg picture where the mass represents the spring constant. The thermal time correlation function at an inverse temperature $\beta$ of the position operator of such as oscillator is given by:

$$
W\left(t, t^{\prime}\right)=\frac{\cos \left(\omega\left(t-t^{\prime}+i \beta / 2\right)\right)}{2 \omega \sinh (\omega \beta / 2)}
$$

which is precisely the expression derived above with $\beta=2 \pi$. This simple computation gives a quantitative support of the relations between Hawking effect in de Sitter and Unruh effect in a flat spacetime as in [12, 13].

We can also invert the transformation (11). Indeed, using the completeness of the radial modes in eqs. (10) we can express the ambient field $\widehat{\Phi}$ as

$$
\widehat{\Phi}(R, y)=\int_{\frac{(d-1)^{2}}{4}}^{\infty} d \lambda \theta_{\lambda}(R) \widehat{\varphi}_{\lambda}(y)
$$

and consequently obtain the following decomposition of the Wightman function

$$
\begin{aligned}
& \left\langle\Omega\left|\widehat{\Phi}(R, y) \widehat{\Phi}\left(R^{\prime}, y^{\prime}\right)\right| \Omega\right\rangle=\int_{\frac{(d-1)^{2}}{4}}^{\infty} d \lambda \int_{\frac{(d-1)^{2}}{4}}^{\infty} d \lambda^{\prime} W_{\lambda, \lambda^{\prime}}\left(y, y^{\prime}\right) \theta_{\lambda}(R) \theta_{\lambda^{\prime}}\left(R^{\prime}\right)= \\
& =\int_{\frac{(d-1)^{2}}{4}}^{\infty} d \lambda \theta_{\lambda}(R) \theta_{\lambda}\left(R^{\prime}\right) W_{\lambda}\left(y, y^{\prime}\right) .
\end{aligned}
$$

This formula allows to express the restriction of the ambient field $\widehat{\Phi}$ to a fixed leaf $R=R^{\prime}$ as a superposition of Klein-Gordon fields in the respective Euclidean vacuum,

$$
\left\langle\Omega\left|\widehat{\Phi}(R, y) \widehat{\Phi}\left(R, y^{\prime}\right)\right| \Omega\right\rangle=\int_{\frac{(d-1)^{2}}{4}}^{\infty} d \lambda\left|\theta_{\lambda}(R)\right|^{2} W_{\lambda}^{(E)}\left(y, y^{\prime}\right) .
$$

The so-obtained Källen-Lehmann type of expansion has a weight in the square mass parameter $\lambda$ given by the density of states per unit spectrum per unit volume of the self adjoint operator $-R^{2}\left(-\partial_{R}^{2}-\frac{d}{R} \partial_{R}+M^{2}\right)$.

These results hold true in any dimension and can actually be generalised to other manifolds. For instance, by the same methods, one can decompose a $d+1$-dimensional Anti de Sitter $(\mathrm{AdS})$ spacetime into a $d$-dimensional Minkowski spacetime and a 1-dimensional Minkowski leaf parametrised by a radial coordinate $R$. In a sense this generalises the geometrical basis of the AdS/CFT correspondence conjecture [1] where one describes the Minkowski space as a boundary of an AdS manifold (that is $R$ is sent to infinity). These and other results will be discussed elsewhere [15, 16]. 


\section{References}

[1] J. Maldacena, Adv.Theor.Math.Phys. 2 (1998) 231.

[2] E. Witten, Adv.Theor.Math.Phys. 2 (1998) 253.

[3] G. t'Hooft, "Dimensional Reduction in Quantum Gravity", gr-qc/9310026.

[4] L. Susskind, J. Math. Phys. 36 (1995) 6377.

[5] G. W. Gibbons, S. W. Hawking, Phys. Rev. D 10 (1977) 2378.

[6] T. S. Bunch, P. C. W. Davies, Proc. R. Soc. Lond. A 360 (1978) 117.

[7] J. Bros, J.-P. Gazeau, U. Moschella, Phys. Rev. Lett. 73 (1994) 1746.

[8] J. Bros, U. Moschella, Rev. Math. Phys. 8 (1996) 324

[9] R. F. Streater, A. S. Wightman, PCT, Spin and Statistics, and all that, W.A. Benjamin, New York (1964).

[10] J. Bros, H. Epstein, U. Moschella, Commun. Math. Phys. 196 (1998) 535.

[11] R. Wald, Quantum Field theory in Curved Spacetime and Black Hole Thermodynamics, The University of Chicago Press (1994).

[12] M. Bertola, Effetti termici della quantizzazione in uno spazio-tempo curvo, Thesis, University of Milan (1995).

[13] S. Deser, O. Levin, Phys. Rev. D 59 (1999) 064004.

[14] H. Bateman, Higher Transcendental Functions, Vol. I and Vol. II, McGraw-Hill, New York (1954).

[15] M. Bertola, J. Bros, V. Gorini, U. Moschella, R. Schaeffer, in preparation.

[16] M. Bertola, J. Bros, U. Moschella, and R. Schaeffer, "AdS/CFT correspondence for $n$-point functions," hep-th/9908140. 